\title{
Hallo! Voulez vous luncher avec moi hït? Le "code switching" dans la communication par SMS
}

\author{
Simona Pekarek Doehler (Neuchâtel)
}

\begin{abstract}
Within the last two decades, text messaging by means of SMS has become a central tool of communication around the globe. The use of more than one language for composing a message is wide spread, but, to this day, is relatively underrepresented in research. This paper presents an analysis of the plurilingual nature of SMS communication in Switzerland with the limelight on the forms and functions of code-switching within a set of 345 messages, base language of which is French. Results show that SMS users regularly exhibit code-switching even if they are not members of a bilingual speech community. Code-switching most frequently consists of inserts, i.e. embeddings of single items or combinations of items within a message composed in another language, and this typically involves (only) a limited range of routinized expressions. While English is the most frequently used language for codeswitching, German, Swiss German, Spanish and Italian are also recurrent, the latter two being particularly associated with terms of endearment. Code-switching regularly highlights the expression of actions that have a strong interpersonal (phatic) focus, such as greetings, goodbyes or thanks. It elucidates the expressive character of the messages, and is also associated with the expression of affection. The specificity of the plurilingual SMS repertoire is discussed in the paper's conclusion.
\end{abstract}

\section{$1 \quad$ Introduction}

Depuis l'envoi du premier message en 1992, la communication par SMS a vécu une véritable explosion. D'après de récentes estimations, plus de quatre billions de messages auront été envoyés au cours de l'année 2010 (Global-Key Statistics, 2010). Une tendance croissante se profile à l'horizon. Les 'textos' (terme souvent utilisé pour renvoyer aux messages SMS) sont devenus un instrument central de communication et de socialisation au travers des cinq continents. Les pratiques du SMS sont multiples - allant des utilisations privées aux emplois professionnels, de pratiques uni- à celles bi- et plurilingues. La co-présence de deux ou plusieurs langues au sein d'un seul et même message est loin d'être une exception et se présente sous des formes variées ( $\mathrm{s} . \mathrm{a} .=$ suisse allemand; all.= allemand; fr.= français; angl.= anglais):

(1) Hallo! Voulez vous luncher avec moi hüt?

$$
\text { ((s.a./all.)) ((angl.-fr.)) ((s.a.: 'aujourd'hui')) }
$$

(2) mon chouchou, comment ca va? tu as bien dormi? je t'aime très très fort

...... hesch hüt mol en freii minute für mi?

ILY

((s.a.: 'est-ce que tu as une minute de libre aujourd'hui'))

((angl: I love you $))$ 
(3) Ça va bien et toi?:-o jap das isch guud! Wänd ja das du

$\begin{array}{lll} & (\text { (s.a: 'c'est bon! } & \text { On veut que tu } \\ \text { au öbis chasch trinke :-) } & \text { grüeßli } & \text { CU 2morrow } \\ \text { puisses boire quelque chose } & \text { salutations')) } & ((\text { angl.: see you tomorrow) })\end{array}$

Toutefois, la nature plurilingue de la communication par SMS n'a à ce jour attiré l'attention de la recherche que de façon marginale.

D'une part, la tradition de recherche linguistique s'intéressant à la communication plurilingue a principalement interrogé les échanges en face-à-face et les communautés bilingues (cf. pt. 2 infra), et ce n'est que récemment que la communication médiatisée par les nouvelles technologies (emails, chats) a attiré l'attention de chercheurs s'intéressant aux choix et aux alternances entre différents codes (voir p.ex. le volume édité par Danet/Herring 2003). D'autre part, les études linguistiques sur la communication par SMS se sont fortement centrées sur les fonctions sociales des échanges SMS et les propriétés orthographiques de celles-ci (voir Thurlow/Poff, à paraitre, pour une vue d'ensemble). Par contre, les dimensions plurilingues de ce moyen de communication globalisé ont jusqu'ici été traitées de manière très incomplète.

Une idée répandue veut que l'«autre» langue présente dans la communication par SMS à travers le monde soit l'anglais, cette présence s'expliquant par la nature 'cool' de cette langue (Crystal 2008). Selon une autre idée, l'alternance codique serait motivée par des raisons d'économie, dans le sens où les utilisateurs, notamment bilingues, auraient tendance à raccourcir la longueur des messages par le choix des lexèmes les plus courts entre leurs deux langues (Bautista 2004). Mais la réalité de l'alternance codique dans la communication par SMS n'est-elle pas plus complexe? Si, depuis l'étude classique de Blom/Gumperz (1972), les travaux d'orientation sociolinguistique, et en particulier ceux émanant de l'analyse conversationnelle, ont révélé la nature multifonctionnelle de l'alternance codique sur le plan social et interactif, mettant en jeu les dimensions identitaires, interpersonnelles, référentielles et organisationnelles des échanges verbaux, pourquoi cela serait-il différent dans la communication par SMS?

L'étude présentée ici vise à contribuer à une meilleure connaissance de la nature plurilingue de la communication par SMS. Elle interroge les formes et les fonctions de l'alternance codique dans un corpus de 345 messages dont la langue principale est le français et qui font intervenir au moins une autre langue à côté du français. Ces messages font partie d'un large corpus recueilli dans le cadre du projet international sms4science (Stähli/Dürscheid/Béguelin, ici même; Dürscheid/Stark 2011; v. aussi www.sms4science.ch). L'étude se penche sur les questions suivantes:

- Quelles sont les langues en présence?

- Quelles sont les fonctions des alternances entre différentes langues (ou codes) au sein des messages envoyés?

- Peut-on constater une dominance de l'anglais sur d'autres langues?

- Existe-il des comportements types dans la manière dont les utilisateurs de SMS changent de langue?

- L'alternance codique dans les SMS relève-t-elle des pratiques de sujets bilingues au sens classique du terme ou se retrouve-t-elle également chez des sujets qui, en principe, ne seraient pas considérés comme tels?

Dans ce qui suit, je présenterai d'abord quelques éléments cadres émanant de la recherche sur l'alternance codique dans la communication en face-à-face (pt. 2) et discuterai l'état de la recherche sur la dimension plurilingue de la communication par SMS (pt. 3). J'exposerai ensuite la nature du corpus étudié et les défis méthodologiques qui en découlent (pt. 4). Sur 
cette base, je proposerai une analyse de l'alternance codique (pt. 5) destinée à identifier ses formes les plus récurrentes et ses principales fonctions dans le corpus SMS. Je conclurai par une discussion des résultats (pt. 6) et des implications pour la recherche (pt. 7).

\section{L'alternance codique et le «parler bi- ou plurilingue»}

Cette section est consacrée à une présentation des principaux types d'alternance codique identifiés dans la recherche antérieure (2.1) et de ses fonctions communicatives (2.2).

\subsection{Code-switching, insertion, alternation, emprunt}

Depuis les travaux fondateurs de Gumperz (1982) et de Poplack (1980), l'alternance codique fait l'objet d'un vif débat portant sur ses dimensions tant grammaticales (quelles sont les contraintes syntaxiques qui régissent l'alternance?) que socio-interactionnelles (quelles sont les fonctions de l'alternance?). On distingue généralement plusieurs types de code-switching, et la terminologie y relative fluctue quelque peu. Dans cet article, j'utiliserai le terme d'alternance codique (et le terme anglais plus répandu de «code-switching») comme hypéronyme pour renvoyer à l'usage de deux ou plus de deux codes au sein d'un même épisode conversationnel (cf. Auer 1998).

La littérature distingue d'une part entre (i) les alternances inter-phrastiques et (ii) les alternances intra-phrastiques (parfois le terme de code-mixing est réservé pour ces dernières; Muysken 2000). D'autre part, sont différenciés les types suivants:

(a) le terme d"insertion' (d'après Auer 1996 et Muysken 2000, appelé antérieurement 'transfert' par Auer 1984) renvoie à l'insertion ponctuelle du matériel d'une langue x (pouvant consister en un morphème, en un item lexical ou en constituants plus grands, p.ex. syntagme de type nominal, prépositionnel ou adverbial) dans un énoncé produit en langue y. L'insertion est donc un phénomène intra-phrastique et correspond à un emboîtement ('embedding' d'après Myers-Scotton 1992) d'une unité d'une langue x dans des structures en langue de base y.

(b) le terme d"alternation' renvoie au changement entre deux structures de deux langues différentes; il s'agit typiquement de changements entre tours ou énoncés (mais Muysken 2000 réserve le terme pour des alternances intra-phrastiques). L'alternation correspond donc à une juxtaposition sans emboîtement de deux différentes langues de base - soit sur le plan intraphrastique (p.ex. deux propositions), soit sur le plan inter-phrastique. ${ }^{1}$

(c) Le terme d"emprunt' ('borrowing') renvoie à l'adaptation d'un matériel lexical aux structures morphologiques, syntaxiques et habituellement phonologiques de la langue d'accueil. L'emprunt, phénomène intra-phrastique, peut être spontané ou sédimenté.

Pour illustrer ces distinctions, revenons brièvement aux exemples (1) et (2), reproduits ici comme (1') et (2'):

Hallo! Voulez vous luncher avec moi hüt?

$$
\text { ((s.a./all.)) ((angl.-fr.)) ((s.a.: 'aujourd'hui')) }
$$

\footnotetext{
${ }^{1}$ Dans la distinction que Auer fait entre code-switching et insertion (terme adopté en 1996 qui remplace le terme 'transfert', proposé en 1984 et qui prêtait à confusion), le code-switching correspondrait à un "changement de la langue-de-l'interaction", et donc à une 'alternation', alors que l'insertion renverrait à "une unité - au minimum un morphème -, qui ne remet pas en cause la langue-de-l'interaction" (1996: 219). Par ailleurs, sur le plan intraphrastique, Muysken (2000) ajoute la catégorie 'congruent lexicalization' pour renvoyer à des cas où deux langues partagent la même structure grammaticale qui peut être remplie par des éléments lexicaux relevant de l'une ou de l'autre langue (comme dans l'exemple suivant, cité par Muysken: Bueno in other words el flight que sale da Chicago around three o'clock, qui pourrait être interprété comme un mélange d"insertion' et d'alternation'.
} 
Dans cet extrait, 'hüit' représente une insertion en suisse allemand, emboîtée dans une structure phrastique dont la langue de base est le français, et y occupant la place d'un complément de temps. 'Luncher' est un emprunt, probablement spontané, morphologiquement adapté à la structure de la langue de base et syntaxiquement placé au bon endroit au sein de cette structure. 'Hallo', d'origine allemande ou suisse-allemande, par contre, est plus difficile à classer. Bien qu'il s'agisse d'un lexème isolé, celui-ci ne relève pas d'une insertion, car il n'y a pas de structure emboîtante. On pourrait le considérer come un code-switching monophrastique (mais qui est différent du du tag-switching; Poplack 1980), le 'hallo' en luimême ayant valeur d'énoncé/acte. D'après Muysken (2000), il y aurait une frontière floue ('transition zone') entre insertion et alternation, notamment dans le cas de tels items isolés (pour des problèmes de classification voir également [Oesch]-Serra 1992).

L'extrait (2') montre un cas typique d'alternation entre des énoncés successifs en différentes langues de base, le locuteur passant du français à l'allemand puis à l'anglais (au travers de l'abréviation ILY).

(2') mon chouchou, comment ca va? tu as bien dormi? je t'aime très très fort

...... hesch hüt mol en freii minute für mi? ILY

((s.a.: 'est-ce que tu as une minute de libre aujourd'hui')) ((angl.: I love you))

\subsection{Le fonctionnement socio-interactif de l'alternance codique}

Dans son ouvrage de référence sur la communication bilingue, Auer (1984) définit l'alternance des langues comme «locally functional uses of two alternate languages in an interactional episode» (p. 7). Deux éléments en particulier méritent d'être soulignés dans cette définition. Premièrement, l'alternance est fonctionnelle dans la mesure où elle accomplit un travail de signalisation; à ce titre, elle est porteuse de significations. Elle représente un indice de contextualisation ${ }^{2}$ (Gumperz, 1982) dans la mesure où elle sert à signaler les orientations des participants les uns envers les autres, à indexer des dimensions sociales et contextuelles de leur rencontre (Auer 1984; Li 2002). Deuxièmement, la fonctionnalité de l'alternance codique est définie localement. Elle n'est pas simplement le reflet direct de valeurs (de prestige, d'appartenance, etc.) attachées à telle ou telle langue ou variété, mais se configure au sein même de l'interaction, au fil de l'organisation séquentielle des conduites mutuelles des interactants. Comme le souligne Auer:

«The proper locus at which semantic values may be assigned to the codes are the very same situations in which language juxtapositions is used for communicative purposes» (1984: 92).

Il s'ensuit que le fonctionnement de l'alternance codique est éminemment lié à son contexte local d'occurrence. Certes, les mêmes éléments de communication ne seront pas interprétés de la même manière par des participants ayant des présupposés socioculturels non identiques. Mais les mêmes éléments de communication ne seront pas non plus interprétés de la même manière selon leur emplacement dans le déroulement de la conversation.

Cette position en matière d'alternance codique, qui se matérialise au travers des travaux d'Auer (1984, 1996), de Li Wei (1998, 2002), de Gafaranga (2005) et d'autres, prend le contre-pied des interprétations dites 'essentialistes' qui veulent que le choix de la langue reflète d'une manière plus ou moins directe des valeurs identitaires et sociales externes aux pratiques communicatives. Les travaux cités réfutent l'idée de significations types

\footnotetext{
2 La notion d'indice de contextualisation renvoie, d'après Gumperz (1977: 199), à «any aspect of the surface form of utterances which, when mapped onto message content, can be shown to be functional in the signaling of interpretive frames».
} 
relativement stables attachées aux choix des langues pour souligner «la configuration locale, collective des ressources plurilingues, de leurs valeurs et de leurs effets» (Mondada/Pekarek Doehler 2003: 99/100). Le focus analytique est donc mis sur la manière dont la signification du code-switching «is brought about by speakers through the very act of code-switching» ( $\mathrm{Li}$ Wei 1998: 170).

Aussi les études empiriques sur l'alternance codique ont-elles montré des emplois et des fonctions variés: marquer l'emphase ou le point culminant d'une histoire (Sebba/Wootton 1998), accroître le potentiel référentiel, indexer l'appartenance de l'événement relaté à un domaine d'expérience ou à un contexte culturel donnés (Lüdi/Py 2003; Auer 1984), marquer un changement de topique ou de structure de participation (Auer 1984), indiquer une appartenance communautaire (Lüdi/Py 2003; Sebba/Wootton 1998), délimiter un segment de discours (p.ex. un discours rapporté, un commentaire métadiscursif, une séquence latérale; Auer 1984; Lüdi/Py 2003), etc.

A travers l'ensemble des travaux cités, l'alternance codique apparaît comme une ressource pour la production de sens dans et par l'interaction communicative. Elle relève d'un «parler bilingue» (Lüdi/Py 2003) ou plurilingue, pouvant en outre manifester la compétence plurilingue du ou des locuteurs (Auer 1996; Lüdi 2004).

\section{La communication par SMS: une communication plurilingue?}

De manière peut-être surprenante, la riche tradition de recherche sur l'alternance codique dans la conversation bi- et plurilingue est jusqu'ici restée sans influence notable sur l'analyse de la communication par SMS. L'investigation linguistique dans ce domaine s'intéresse de manière prioritaire aux fonctions sociales des échanges SMS, à la variation des pratiques en fonction de facteurs tels que l'âge, le genre des utilisateurs et le contexte socioculturel, et aux transformations et adaptations que subit l'orthographe au sein de ce medium de communication. Certaines de ces transformations ont été expliquées par des contraintes pratiques du téléphone portable, dont en premier lieu l'utilisation d'un tout petit clavier et la limite du nombre de caractères par message (le standard général étant de 160 caractères) ${ }^{3}$. Thurlow (2003) identifie trois maximes sociolinguistiques qui pèseraient sur la pratique du SMS, laissant des traces sur son formatage linguistique:

(i) condensation et rapidité, qui se traduiraient notamment par des abréviations (p. ex. 'c qu' pour la formule d'adieu 'see you') et la réduction de la ponctuation;

(ii) le marquage d'emphase et d'états émotionnels par exemple par l'utilisation d'émoticônes (tels les smileys ';-)') ou l'utilisation de majuscules et la multiplication de marqueurs de ponctuation (p.ex. !!!???);

(iii) l'approximation phonologique (p.ex. 'je c' pour 'je sais').

Alors que ces éléments rendent compte de certaines adaptations spécifiques du langage dans la communication par SMS (pour le français voir Anis 2002, Cougnon 2008, et

\footnotetext{
${ }^{3}$ Possiblement, l'effet des contraintes pratiques sur la rédaction des SMS change à l'heure actuelle avec l'avènement des smartphones, qui remplacent progressivement les anciens portables. A titre anecdotique, voici le témoignage d'une utilisatrice (commentaire personnel de Cécile Petitjean): «A mon sens, ces contraintes techniques jouent un rôle important dans les pratiques des utilisateurs de sms. D'autant plus en ce moment, où les anciens portables sont progressivement remplacés par des smartphones, qui présentent des fonctionnalités très différentes dans la rédaction des messages. Personnellement, depuis que j'en ai un, avec un clavier tactile (qui évite de devoir appuyer plusieurs fois sur une même touche pour obtenir la lettre désirée), je me rends compte que je rédige des sms, d'une part, plus longs, et, d'autre part, en utilisant un nombre bien moindre d'abréviations (et avec beaucoup plus de signes de ponctuation). Ma pratique du sms se rapproche considérablement de ma pratique de l'email.»
} 
Fairon/Klein/Paumier 2006), la question demeure de savoir si, et dans quelle mesure, elles peuvent contribuer à expliquer l'alternance des langues dans les messages SMS.

Les études s'intéressant à la coprésence, au sein des messages SMS, de deux ou plusieurs langues sont rares. Elles prennent typiquement comme point de départ la pratique de sujets bilingues au sens classique du terme, c'est-à-dire de sujets qui pratiquent régulièrement plus d'une langue. Centrées sur les contextes culturels multilingues, ces études interrogent pour la plupart l'utilisation de l'anglais en combinaison avec une autre langue, et s'intéressent souvent à la fréquence des deux langues et à l'effet de leur coprésence sur la longueur des messages.

Dans une étude sur des sujets bilingues anglais-tagalog, Bautista (2004) suggère que le multilinguisme augmenterait l'efficacité de la communication par SMS, en permettant à des sujets bilingues de choisir les lexèmes entre les deux langues en fonction de leur longueur, avec une préférence pour les lexèmes plus courts, ce qui leur permettrait de réduire le nombre de caractères par mot/message. Cette explication, qui ramène l'alternance codique à une seule fonction, contraste fortement avec ce que nous savons sur la complexité du fonctionnement de l'alternance codique dans la communication en face-à-face (cf. pt. 2 supra). Aussi l'hypothèse de Bautista a-t-elle été remise en question par plusieurs études empiriques sur les messages SMS.

Dans deux études directement destinées à tester l'hypothèse de Bautista (2004), Carrier et Benitez (2010) réfutent l'idée de l'économie comme critère explicatif de l'alternance codique. Une première étude analyse la longueur de messages authentiques recueillis auprès de 26 étudiants d'université bilingues anglais-espagnol en Californie (565 messages au total, avec 141 messages comprenant les deux langues). Les résultats montrent que les messages comprenant des alternances codiques sont en moyenne plus longs que les messages qui n'en contiennent pas. La seconde étude, de nature expérimentale, implique 16 paires d'étudiants bilingues anglais-espagnol et 22 paires d'étudiants monolingues anglais dans un jeu élaboré pour encourager l'efficacité de la communication par SMS. L'étude ne révèle aucune différence significative en termes de longueur (nombre de caractères et de mots) entre les messages échangés parmi les bilingues et ceux échangés parmi les monolingues.

Deumert et Masinyana (2008) présentent des résultats qui vont eux aussi à l'encontre de l'hypothèse de Bautista (2004), bien que leur étude n'ait pas été directement élaborée pour tester cette hypothèse. Les auteurs ont étudié les messages de 22 bilingues sud-africains anglais-isiXhosa âgés entre 18 et 27 ans. Ils montrent que les messages bilingues contiennent en moyenne non pas moins mais plus de caractères que les messages monolingues. Ils mentionnent également que les messages bilingues de leur corpus contiennent en moyenne plus de mots que les messages unilingues en isXhosa (ce qui s'explique par la nature agglutinante de cette langue), mais un mot de moins que les messages unilingues en anglais. Tout comme les deux études effectuées par Carrier et Benitez (2010), l'analyse de Deumert et Masinyana (2008) réfute les contraintes d'économie et d'efficacité comme facteur expliquant l'utilisation de deux langues dans un même message SMS.

De manière intéressante, les pratiques SMS plurilingues documentées jusqu'ici dans la littérature diffèrent selon les contextes culturels. Dans son analyse des pratiques SMS d'étudiants au Kuwait, Haggan (2007) explique la coprésence récurrente de l'anglais et de l'arabe dans les messages SMS par le fait que, durant longtemps, le clavier sur les téléphones mobiles était disponible uniquement en avec un alphabet latin et non pas arabe. Chiluwa (2008) montre qu'au Niger, l'Anglais nigérien et d'autres langues indigènes sont évitées au sein de la communication par SMS en faveur de l'anglais standard. Deumert et Masinyana (2008) constatent des différences marquées dans la façon dont l'anglais et le isiXhosa sont utilisés en Afrique du Sud. Alors que les messages en anglais montrent les caractéristiques considérées comme typiques du langage SMS (abréviations, orthographe non-standard, etc.), 
liées aux trois maximes sociolinguistiques postulées par Thurlow (2003, voir supra), les messages en isiXhosa révèlent une utilisation tout à fait standard de cette langue, mais intègrent des code-switches fréquents en anglais. Deumert et Masinyana (2008) suggèrent que le caractère non-standard des messages en isiXhosa est affiché non pas par la déviation en matière d'orthographe, mais par la pratique du code-switching.

Les études citées attestent d'une grande diversité des pratiques plurilingues de sujets bilingues. Elles ne nous renseignent toutefois pas sur les pratiques de sujets qui ne seraient pas classiquement considérés comme bilingues, ni sur la nature précise des alternances codiques. Cougnon (à paraitre), par contre, dans une étude sur un corpus de messages SMS recueillis en Belgique et dont la langue de base est le français, offre une série d'observations sur les formes et les fonctions du code-switching. L'auteur identifie dans les pratiques du code-switching une claire dominance quantitative de l'anglais sur d'autres langues. Elle suggère que l'anglais joue de manière récurrente le rôle d'intensificateur lors d'expression de sentiments, alors que les langues latines seraient utilisées en raison de leurs connotations affectives.

Il peut être intéressant de noter que le choix de la langue et l'alternance codique ont fait l'objet, au cours de la décennie passée, d'une série d'études sur la communication médiatisée par ordinateur. Plusieurs études sur la communication asynchrone par email (p.ex. Durham 2003; Goldbarg 2009; Ho 2006; Warschauer/El Said/Zohry 2002) se sont centrées sur des sujets bilingues dans divers contextes linguistiques et culturels où la langue locale (suisseallemand/allemand, espagnol, chinois) se combine avec l'anglais. Prises ensemble, ces études révèlent un lien entre choix de la langue et objectif, voire thème de l'échange: les langues avec lesquelles les locuteurs s'identifient le plus seraient davantage associées à l'expression de pensées personnelles et à la discussion de thèmes d'un certain degré d'intimité (ce qui rejoint en gros les travaux classiques de Gumperz); l'anglais serait par contre souvent utilisé pour traiter de thèmes liés au monde des ordinateurs.

En somme, à l'état actuel de la recherche, nous disposons d'une série de résultats sur les associations tendancielles entre choix de la langue, thèmes et nature des échanges médiatisés par ordinateur. Nous disposons également de résultats qui montrent que les pratiques plurilingues dans la communication par SMS chez de sujets bilingues diffèrent selon les contextes culturels, avec des préférences variables pour l'une ou l'autre des langues en question, et des taux d'occurrence variables de celles-ci. Par contre, les formes et les fonctions précises de l'alternance codique dans la communication SMS, notamment dans des contextes culturels à dominance non-bilingue (comme c'est le cas p.ex. pour la Suisse Romande), sont restées largement inexplorées. La présente étude cherche à contribuer à combler cette lacune.

\section{Données et défis méthodologiques}

\subsection{Données}

Cet article présente une étude exploratoire de l'alternance codique sur la base d'un corpus de 345 messages bi- ou plurilingues qui a été établi par un tri manuel à partir d'une base de données composée de 23'764 messages SMS récoltés en Suisse, dont 4'505 en français, dans le cadre du projet sms4sience (www.sms4science.ch). Le recueil s'est fait par le biais d'un appel à donation ('faites don de vos SMS') diffusé à large échelle, sans aucune présélection des auteurs des messages. Le corpus analysé ici a été établi par Samuel Da Silva, Alexis Ndabihonge et Emanuel Schard dans le cadre d'un travail de séminaire (Da Silva/Ndabihonge/Schard 2009). Il est composé de messages qui partagent les deux caractéristiques suivantes: (a) la moitié au moins du message est composé en français; (b) une autre langue en plus du français est présente. Le corpus n'est pas exhaustif, au sens où il ne 
comprend pas la totalité des messages correspondant à ces critères. Il présente simplement un premier recueil de messages plurilingues extraits de la base de données, comprenant un nombre suffisant de messages pour une analyse exploratoire. Les messages ont été anonymisés et les noms propres y figurant ont été remplacés par la séquence ANON. Les messages sont reproduits ici avec l'orthographe et la ponctuation originales.

La Suisse, bien qu'officiellement plurilingue, est régie par le principe de territorialité: les régions sont - à peu d'exceptions près - majoritairement unilingues. Nous pouvons en déduire que les messages recueillis en Suisse n'ont pour la plupart pas été produits par des personnes appartenant à une communauté bi- ou plurilingue - bien que certains utilisateurs peuvent bien sûre appartenir à une telle communauté. Ce fait se reflète largement dans les messages recueillis, dans la mesure a) où les messages à dominance française ne comportent que rarement des énoncés grammaticaux dans une autre langue (en allemand ou en suisseallemand, notamment) et $b$ ) où les segments produits dans une autre langue se limitent pour la plupart à un inventaire limité de mots isolés ou d'expressions figées (cf. pt. 5 infra). Ces éléments suggèrent que les messages analysés ont été composés pour la plupart par des sujets qui ne sont pas bilingues au sens classique du terme (cf. pts. 5 et 6 infra). Bien qu'il existe des données socio-biographiques concernant les destinateurs d'une partie des messages réunis dans le projet sms4science, ces données ne sont pas encore disponibles à l'heure actuelle pour une analyse systématique. Ce que nous savons à l'heure actuelle est que les messages analysés ici nous ont été envoyés à 2/3 par des personnes jeunes, âgées de 11 à 30 ans.

\subsection{Défis méthodologiques: analyser l'alternance codique dans un corpus de messages SMS}

La recherche existante sur l'alternance codique s'est massivement centrée sur l'analyse d'interactions orales (cf. pt. 2 supra). Les catégories et les procédures analytiques de cette riche tradition de recherche, si elles peuvent enrichir notre approche de la communication par SMS, ne sont pas transférables telles quelles aux données SMS écrites. Les caractéristiques du corpus SMS étudié ici soulèvent plusieurs défis méthodologiques.

Premièrement, le corpus est composé de messages isolés; il ne permet pas de retracer des échanges de messages. Cette propriété impose d'importantes limites à l'analyse de l'alternance codique, rendant notamment impossible une analyse séquentielle qui se fonderait sur l'enchaînement des tours de parole/des messages. Nous n'avons pas accès au tour qui précède l'alternance codique, ni au tour qui suit, ni encore au contexte interactif plus large. Cela signifie, notamment, que nous ne pouvons pas retracer la manière dont le locuteur suivant traite l'alternance codique, ni comment celle-ci s'insère dans l'échange en cours

Deuxièmement, toujours en raison de la nature isolée des messages de notre corpus, il est impossible de déterminer la langue de l'interaction; seule la langue du message peut servir comme point de référence pour l'analyse.

Troisièmement, la nature écrite du corpus étudié pose le problème de l'homographie de mots qui appartiennent à différentes langues. A titre d'exemple, le mot chaos s'écrit de manière identique en français, en allemand et en anglais. Il est donc impossible d'identifier si telle ou telle occurrence de ce mot relèvent ou non d'une alternance codique. Le même problème se pose pour des utilisations non standard d'abréviations, telles le 'n8' qui peut traduire l'allemand 'n-acht' ('Nacht'), l'anglais 'n-(e)ight' ('night') ou encore le français 'n-huit' ('nuit').

Quatrièmement, la nature écrite du corpus fait que la production langagière n'est accessible pour l'analyste qu'en tant que produit post hoc, et non pas en tant que processus en temps réel. Par conséquent, des observables comme les hésitations et les auto-réparations ne sont pas disponibles, alors que celles-ci ont fourni dans les travaux sur l'interaction orale des indices 
clés pour différencier par exemple l'alternance codique, et la formulation transcodique (stratégie pour surmonter une lacune linguistique).

Face à ces difficultés, les choix méthodologiques entrepris ici se résument de la façon suivante:

1. Les analyses ont été menées de manière étique (i.e. en suivant l'interprétation du chercheur), faute d'accès aux interprétations que les interlocuteurs eux-mêmes font des alternances codiques, telles qu'elles seraient analysables à travers l'enchaînement séquentiel des messages. Elles se centrent sur les caractéristiques récurrentes de l'alternance codique aux niveaux de ses formes et de ses fonctions.

2. La langue du message a servi de point de repère pour identifier une alternance codique; ont été retenus dans le corpus les messages rédigés majoritairement en français, soit des messages dont la langue de base est le français et ceux qui font intervenir des segments dont la langue de base est une autre langue, mais où les segments en français dominent.

3. Les messages contenant des items dont la provenance est incertaine en raison de leur caractère homographique n'ont pas été inclus dans le corpus (p.ex. 'chaos').

Nous verrons plus loin (pt. 5) que la nature même des alternances codiques récurrentes, et notamment la fréquence de formes-types d'alternance, permet en principe d'exclure l'idée que l'alternance relèverait de mécanismes compensatoires liés à des lacunes linguistiques.

\section{$5 \quad$ Analyses}

\section{1 'Alternation' et 'insertion': quelques observations générales}

La première caractéristique notable du corpus des 345 messages contenant des alternances codiques est que les alternances sont le plus souvent des mots isolés ou des expressions figées. Il s'agit soit d'insertions ponctuelles d'expressions provenant d'une autre langue dans des énoncés dont la langue de base est le français, soit d'alternances d'un type spécifique que nous traiterons comme relevant ni de la catégorie 'insertions' ni de la catégorie 'alternations'; celles-ci consistent en des formules figées toute faites comme 'hello' ou 'a domani' qui forment un énoncé en soi et ne sont donc pas insérées dans des structures grammaticales produites en une autre langue (raison pour laquelle on pourrait les traiter comme 'alternation'), mais qui semblent être utilisées comme des chunks (ou: expressions figées) non-analysés grammaticalement (ce qui les différencie des cas courants d'alternation'). Les alternances de type 'alternation', entre segments grammaticaux en différentes langues de base, sont par contre rares.

Les exemples (4) et (5) respectivement représentent les deux cas typiques de code-switching figurant dans le corpus: a) l'insertion d'un lexème dans une structure en français; b) l'utilisation d'un élément isolé (lexème ou expression figée) à valeur d'énoncé/acte (ici, d'adieu).

(4) On rentre now et on découvre votre très jolie et gentille carte de remerciement!Merci bcp!Merci pr dimanche,ca m'a fait plaisir!A tout bientot,gros bisous

(5) Super merci.a domani

L'exemple (6) montre le cas, plus rare dans nos données, d'une alternance entre deux segments grammaticaux en deux langues de base différentes (type 'alternation'):

(6) Je t'appelle quand j'ai fini le schwitzertütsch. Ich ha dir gärn müïsli. 
Parmi les 'alternations', on retrouve régulièrement la présence du suisse-allemand (ex. 6 supra), parfois de l'allemand standard (ex. 7):

(7) Ist gut, j'ai déjà acheté quelque chose,

((all: 'c'est bon'))

wenn spaghetti und tomatensauce okay sind.

((all: 'si des spaghettis et sauce tomate est o.k.'))

Enfin, l'emprunt spontané, qui se traduit par l'adaptation ad hoc de formes empruntées à une autre langue à la structure morphosyntaxique du français, fait figure d'exception:

(8) Hallo! Voulez vous luncher avec moi hüt?

(9) Pfff il est tjrs surbooké! ;) A domani alors becs

Les alternances consistant en une insertion (ex. 4), et celles composées d'un seul mot ou d'une expression figée à valeur d'énoncé/acte (ex. 5), montrent un éventail limité de formes et de fonctions hautement récurrentes (cf. pt. 5.2 infra). Par contre, les alternances entre différentes langues de base, du type illustré sous (6) et (7), qui ne représentent que $12 \%$ des code-switchs dans notre corpus, se présentent sous des formes diversifiées. Il est possible que les messages concernés proviennent pour la plupart de personnes bilingues français-allemand/suisseallemand; clairement, ils sont produits par des personnes dont le répertoire linguistique dans la langue autre que le français dépasse l'inventaire des formes types récurrentes que nous repérons pour les insertions et des formules figées (voir pt. 5.2 infra). Leur analyse approfondie présupposerait que l'on puisse suivre l'échange des SMS au-delà du message isolé; pour cette raison, elles seront écartées des analyses qui suivent (mais voir pt. 5.5 infra).

\subsection{Un inventaire limité de formes routinisées d'alternance codique}

Le tableau suivant résume les items qui sont le plus fréquemment exprimés dans une langue autre que le français dans les données; il indique le nombre de leurs occurrences ainsi que le nombre d'occurrences de leur 'équivalent' en français et dans d'autres langues:

\begin{tabular}{|l|l|l|}
\hline $\begin{array}{l}\text { Alternances codiques les plus } \\
\text { fréquentes et nombre } \\
\text { d'occurrences }\end{array}$ & $\begin{array}{l}\text { Nb.d'occurrences de } \\
\text { l'équivalent en français }\end{array}$ & $\begin{array}{l}\text { Nb. d'occurrences de } \\
\text { l'équivalent dans d'autres } \\
\text { langues }\end{array}$ \\
\hline $\begin{array}{l}\text { hey: } 37 \\
\text { hello: } 12\end{array}$ & salut: 28 & $\begin{array}{l}\text { ciao: } 9 \\
\text { hallo: } 1 \\
\text { hola: } 1\end{array}$ \\
hi: 4 & bonjour: 5 & lo siento: 1 \\
\hline sorry: 17 & excuse-moi: 1 & - \\
\hline today: 10 & aujourd'hui: 4 & - \\
\hline now: 7 & maintenant/ m1tenan/ mintnt: 4 & \\
\hline
\end{tabular}

Trois éléments sont à noter au sujet de ce tableau:

(i) Les alternances codiques concernent le plus fréquemment des expressions courtes, consistant en un seul mot et produites typiquement en anglais (mais voir les 9 occurrences du 'ciao').

(ii) Pour tous les cas cités, l'utilisation d'expressions dans une autre langue que le français prédomine sur l'utilisation de l'équivalent français: les excuses sont quasi exclusivement accomplies au moyen de la formule anglaise 'sorry' (17 occurrences vs. un seul 'excuse-moi'); les salutations sont souvent effectuées au moyen de diverses formes en anglais ou de l'italien 'ciao' (64 occurrences en une langue autre que le français, dont 53 en anglais, 9 en italien, 1 en allemand et 1 en espagnol vs. 33 occurrences en français, dont 28 'salut' et 5 'bonjour'); les renvois déictiques au 
maintenant sont pour la plupart effectués par l'anglais 'today' (10 occurrences vs. 4 'aujourd'hui' et ses variantes orthographiques) et 'now' (7 vs. 6 'maintenant' et ses variantes orthographiques).

(iii) Ces éléments apparaissent régulièrement de manière isolée, représentant à eux seuls une alternance codique, comme le montrent les exemples (10) à (15).

(10) Hey!! Commen ca va? T'as vu mon mail? Avec ANON,on se retrouve à 16 h30 à la Mig,o restau tt en-haut. Tu peux venir? Jsui dsl d'te prévenir si tard...

(11) Hello je viens de réaliser que hier on avait rdv ds le train... Sorry!

(12) Un petit pâté de vents d'ouest nous apportera un peu de fraîcheur today.

(13) C'est demain ton vernissage ou today? Pke jsuis à gnève ce soir mais pas demain

(14) Coucou vava Ça va? Alors vs etes déjà à la fete? On descend now! Gro bisous

(15) Je pars now, à toute, bisous mon cœur

Les propriétés citées sous (i) à (iii) montrent un emploi typé de formules notamment anglaises. Les 'today' et les 'now' relèvent d'alternances codiques de type insertion (ex. 12-15): elles sont 'emboitées' dans des structures phrastiques en français. Les occurrences des expressions isolées à valeur d'énoncé/acte de type 'hello', comme dans (10) et (11), alternent avec des emplois où celles-ci sont combinées avec des termes d'adresse en français ou en anglais (p.ex. 'hello l'anglaise', 'hi miss' , 'ciao bella', cf. pt. 5.3 infra). Tous les cas cités portent les traces d'un certain degré de sédimentation dans les données étudiées: ces éléments sont non seulement fréquents, mais ils prédominent sur les équivalents en français. Il est toutefois à noter que les calculs proportionnels ont été effectués à partir d'un sous-corpus qui ne comportait que des messages bi-/plurilingues; il ne s'agit donc pas de proportions absolus pour les messages composés en français. Parmi les éléments cités - mis à part le 'ciao' qui se retrouve sous la forme 'tscho' en français parlé, et le 'miss' également présente en français parlé informel -, aucun ne relève de pratiques courantes dans la communication orale en Suisse francophone 4 . Notons toutefois que le 'ciao' italien est employé dans les SMS exclusivement en position initiale d'ouverture du message, et donc comme forme de salutation, alors que le 'tscho' en français parlé est utilisé tant en ouverture (comme équivalent de 'salut') qu'en clôture d'échange comme formule d'adieu. Les cas cités sont donc différents d'un cas comme 'cool', expression provenant de l'anglais qui est sédimenté en français, notamment informel, sous forme d'emprunt, et qui réapparaît dans les messages SMS (nous en avons recensé 16 occurrences). Contrairement à une expression comme 'cool', la fréquence des expressions 'hello', 'hi', 'hey', 'sorry', 'now' et 'today' - mais aussi 'ciao', avec sa position séquentielle spécifique -, et surtout leur prédominance sur les équivalents en français, sont un trait distinctif des messages SMS. Ceci suggère que ces expressions font partie de routines communicatives spécifiques à la communication par SMS, relevant possiblement d'un répertoire SMS qui puise fortement dans l'anglais. Il n'est pas exclu que les raisons d'économie aient un effet favorable à l'emploi d'équivalents anglais courts (p.ex. 'today', 'now', 'sorry'), notamment pour les formes françaises 'aujourd'hui', 'maintenant' et peut-être aussi 'excuse(-moi)'.

\subsection{L'augmentation de l'expressivité}

Un second élément notable au sujet des données étudiées est que l'alternance codique y est systématiquement associée à un éventail restreint d'actions à caractère notamment phatique et

\footnotetext{
${ }^{4}$ Le Centre National de Ressources Textuelles et Lexicales (www.cnrtl.fr) du CNRS indique que 'ciao' est lexicalisé, à l'écrit, depuis les années 50 et 'miss' l'est tout autant. Je remercie Cecilia Serra d'avoir attiré mon attention à ce fait.
} 
affectif. L'analyse qui en est présentée dans cette section montre que cette alternance a pour effet d'augmenter l'expressivité des énoncés correspondants.

\subsubsection{L'augmentation de l'expressivité I: des actions à fort caractère phatique}

Un éventail d'actions à forte dominance phatique est accompli dans le corpus au moyen d'alternances codiques. Nous avons déjà noté l'utilisation privilégiée de l'alternance codique pour des formules de salutation (ex. 10,11; 16-18) et les excuses $(11 ; 19-20)$ :

(16) Hola! T'es où? Y a moyen de se joindre ce soir? Désolé si tu m'as dit que tu serais occupé et que j'ai zappé... Bisous!

(17) Hallo! Voulez vous luncher avec moi hüt?

(18) Hey there! Oui bien sur passe chez moi plutot en début d'aprem right? J'ai ta robe aussi l'oublie pas haha :)

(19) Hello je viens de réaliser que hier on avait rdv ds le train... Sorry !

(20) Sorry :\$ !mais tétai pa censé savoir en tt cas pa avant 2 semaine au minimum !=p

A côté des salutations et des excuses, l'alternance est également récurrente pour les adieux:

(21) Super merci.a domani

(22) Espagnol la langue! :) les articles j'ai pas du tout le courage, meme pas un! Ah ok, ton oncle est sorti en boite à Yverdon? Ghgh hasta magnana!

(23) ouCou!Dsl 2 répondr k now!Pr le cavalo,o fait,cè la caissièr,é pa ma patron.Ca serai just 1e sortie lé 2.Oui,vè alé au soupé!Domag $\mathrm{k}$ tu soi pa là!Mè $\mathrm{t}$ raconterai (par mail?)! On va alé soupé ché ANON,où ANON travail m1tenan.Hier,g revu ANON,c étè super sympa!On a pu alé boire un ver ensembl..Bonne journée!\& $\boldsymbol{C U} S \boldsymbol{S o o N}$ !

((angl.: see you soon))

Elle est également fréquente pour les félicitations et les vœux de toutes sortes (ex. 24-27) ainsi que pour les remerciements (ex. 28 et 29):

(24) Good good luck pr ton exa!!

(25) Salut Stéph! HAPPY BIRTHDAY TO YOU!! je te souhaite de passer une bonne journée pour ton anni. Gros becs

(26) Auguri monsieur! A bientot pour fete sur Neuch! Baci

(27) Nighty night! Je t'aime, ma belle

(28) Oui très joli venise, vraiment, tu as vu la basilique st-marc et ses mosaiques? Et super glaces! La, arrivés a rome, ss tente, gros orage pas loin, tonne fort. Perce-oreille entrée ds sac couchage, la retrouve pas, m'enerve. Si t'as un moment demain, tu pourrais regarder sur internet si pluie prévue par ici? Grazie mille et bon week a toi aussi! Bises.

(29) Anyway voici ma proposition: mets- moi sur le plan du 1 er janvier à ta place. Vraiment cela me conviens pour ce jour- là! Thanks, A

Enfin, on retrouve régulièrement la formule de politesse 'please' (correspondant au français 's'il te plait', respectivement 's'il vous plait') sous différentes variantes orthographiques:

(30) Oh.... Dis tu peu me fil le num à ANON pliz ?

(31) Hello!!sa va??avec le coupon fondue tu pourrai me donné le dvd casino de ANON bond please!!merci bcp bisou

(32) Demande au autre mé Moi jé pa envi d paié $20 \mathrm{fr} 1$ entre+4bière il me semble que Noel ma di kelke chose pr demain mais je suis pas sur pouré tu lui demandé please 
Contrairement aux éléments comme 'sorry' ou 'now', qui semblent sédimentés dans leur forme anglaise, l'utilisation de l'anglais ou d'autres langues pour l'accomplissement d'actions phatiques alterne régulièrement avec l'utilisation d'expressions en français, comme 'merci' (ex. 31), 'bonne chance' et 'stp'.

Il est à noter que les alternances liés à l'expression d'actions à fort caractère phatique mobilisent un inventaire restreint de formules d'excuse, de salutation, d'adieu, de vœux et de remerciement, consistant en un seul mot ou en une expression figée (comme 'good luck'). Les formules relevant de l'anglais notamment semblent être hautement routinisées, tout particulièrement pour les excuses et les salutations (cf. supra). Par contre, l'anglais alterne avec l'italien et l'espagnol pour l'expression d'adieux, de remerciements et de vœux (ex. 21, $22,2628)$.

Mais quelles sont les fonctions communicatives de ces alternances codiques? On notera d'abord que les expressions utilisées sont parfois plus courtes et parfois plus longues que ne le serait la formulation en français: alors qu'un 'sorry' est plus court qu'un 'excuse' - mais d'une seule lettre -, le nombre de caractères d'un 'thanks' ou d'un 'please' est plus élevé que celui du 'merci' ou du 'stp'. Dans certains cas, le contenu du segment produit sous la forme d'une alternance codique se trouve même dédoublé ou reformulé par une formulation en français (ex. 25), dans une logique contraire à l'économie de l'écriture. Ces propriétés montrent clairement que le critère d'économie n'est pas un facteur explicatif valable de l'alternance codique dans la plupart des cas cités.

Par contre, deux autres propriétés des alternances codiques citées dans cette section nous renseignent sur le travail discursif qu'elles accomplissent. L'association régulière de l'alternance codique à l'accomplissement d'actions à caractère hautement phatique et leur caractère ponctuel (expressions figées, courtes, qui se limitent exactement à l'accomplissement des dites actions) suggèrent que l'alternance codique dispose ici d'une fonction de démarcation de l'action à caractère phatique par rapport au reste du message. L'utilisation de l'alternance dans ces contextes où la gestion de la dimension interpersonnelle est spécifiquement en jeu a pour effet d'augmenter l'expressivité du dire en rendant particulièrement saillantes les actions en question. Cette interprétation est corroborée par le fait que ces alternances sont fréquemment accompagnées de moyens d'emphase typiques pour la communication par SMS (cf. Thurlow 2003), comme les points d'exclamation (p.ex. 16-20, 22, 23, 25, 27), les majuscules (ex. 23, 25) et les émoticônes (ex. 20).

\subsubsection{L'augmentation de l'expressivité II: l'expression de l'affectivité}

L'utilisation de l'alternance codique au service de l'expressivité se retrouve dans un autre cas de figure, à savoir l'expression de l'affectivité. De manière intéressante, plus on glisse vers l'expression de l'affectivité au-delà de la gestion de la dimension phatique, plus l'alternance codique mobilise une panoplie de langues différentes. Tel est le cas des adieux affectifs (ex. 33-36), et tout particulièrement des déclarations d'amour qui, régulièrement, font foi d'adieu (37) ou sont combinées avec une formule d'adieu (38):

(33) C pa grave,j'compren. J'avoue ke jsui bien naze aussi,et j'commence à regretter d'avoir accepté de bosser demain matin:-o La nuit va etr courte...:-S. Besitos

(34) ANON bonjour!ça va?l'initiative des minarettes est horrible!-je sais pas que dire-mais dis moi-tu ha temps semaine prochaine mercredi ou jeudi pour une visite dans le atelier?liebgru $\beta$ - $\mathrm{k}$

(35) Coucou c toujours ok pour 16h40? Smack

(36) Loulou, n'oublie pas le 4 déc. Lieu encore à determiner. Cela va pr toutes, sauf ANON. Buona giornata, baci grandi 
J'ai ton petit cadeau mais si je le laisse à ton bureau il va geler alors te le donne quand je te vois mais je dépose déjà un doux baiser dans ta boite pour te souhaiter un joyeux anniversaire gros calin!I love you

(38) Bijour!! Euh... bonsoir!! :-) Ca va? Bien fini le boulot? Bon souper avec ANON, et tiens moi au courant!! Ti amo mucho!! Besos!

Tout comme les actions à fort caractère phatique, les manifestations de l'affectivité dans les prises de congé sont accomplies par le biais d'un inventaire restreint de formules types. Or, l'avènement de la dimension affective au-delà de la dimension phatique semble se combiner avec une multiplication des langues sources pour l'alternance codique: alors que pour les excuses, les salutations et les félicitations citées plus haut, l'anglais dominait fortement, l'expression de l'affectivité mobilise régulièrement à côté de l'anglais l'italien et l'espagnol, mais aussi l'allemand (ex. 34, voir aussi infra).

\subsection{Les termes d'adresse: des petits noms tendres aux surnoms 'cool'}

Un autre élément fréquemment exprimé au moyen de l'alternance codique sont les apostrophes, c'est-à-dire les termes qui désignent la personne à laquelle le locuteur s'adresse (Riegel et al. 1994). Ceux-ci vont de petits noms tendres à des expressions plus «branchées » de type 'man' ou 'buddy'. Les apostrophes tendres corroborent des observations exposées dans la section précédente, à savoir a) l'association de l'alternance codique à l'expression de l'affectivité, et b) la mobilisation de l'italien et de l'espagnol pour celle-ci, à côté de l'anglais:

(39) eah baby! J'suis trop happy, tu verras comme c'est cool!!! Tèm fort [...]

(40) Amore! C'était tellement génial de te voir, je sais pas comment j'ai pu attendre aussi longtemps! Je suis maso ou bien?...

(41) My little fanouille...tu me manques trop à la folie [...]

(42) Bonsoir a tous! [...] gros poutoux partout les amigos!

(43) Bonne nuit Amore, nous sommes au lit. T'as oublié la bague, j'espère. [...]

Parfois, le terme d'adresse est combiné avec une forme de salutation en français ou dans la même langue que l'apostrophe elle-même, et est alors typiquement placé en début de message:

(44) Buenas noches querido... C'est sympa alors cette soirée à l'aéroport avec Brissouille?^^^ Fais d'beaux reves, bisous doux...

(45) Ciao amore, c'était ta soirée hier (victoire de féfé et du barca)! Trop cool! Je te fais un bec et te souhaite une bonne journée

(46) Ciao bello,tu croyais que jtavais oublié mais non,je pense técrire depuis ce matin,mem si jle fais que maintnt.. J'espère que tu as passé une belle journée,bise

(47) Salut mon cookie chéri ! C'était juste super cette soirée avec ton sourire et ta bonne humeur ! Tu sais quoi ? Je t'aime très fort! (plus que toi :-) ) bonne nuit fait pleins de magnifiques reves comme toi ! ... Je t'aime

Ces apostrophes tendres vont des expressions plus courantes comme 'baby' ou 'amore' à des formulations plus idiosyncrasiques. L'extrait (47) montre un terme d'adresse complexe, produit en deux langues: 'mon' et 'chéri' en français, comprenant le 'cookie' intercalé en anglais. Une autre formulation idiosyncrasique, non-transparente pour un externe, est le 'my little fanouille' de l'extrait (41). Ici, le déterminant possessif et l'adjectif 'little' relèvent de l'anglais, alors que le terme 'fanouille', qui ne se retrouve pas dans les dictionnaires, représente possiblement un surnom «privé», tout en portant les traits orthographiques et morphosyntaxiques typiques du français (notamment la terminaison '-ouille'). Dans ces deux cas, les apostrophes sont composées en mobilisant deux langues. 
On retrouve également l'utilisation de l'alternance codique sous des formes originales pour les apostrophes ludiques, à visée humoristique, relevant possiblement de «blagues privées», comme dans le cas suivant:

(48) Salut les bananitas!! J'voulais savoir si c'était possible que vs fassiez votr séance,ce samedi.J'voulais aller à la ferme,mais ca joue pa pr le paysan...

Alors que certaines des apostrophes produites dans une langue autre que le français semblent relever d'un répertoire intime propre à des couples ou à des amis spécifiques, comme c'est le cas du 'cookie' ou de 'my little fanouille', d'autres, comme 'baby' ou 'amore', mobilisent des expressions quasi internationales dans la mesure où elles sont répandues comme formules couramment utilisées dans le parler informel en plusieurs langues (Eaton/Yarmanova 2009). Cela est systématiquement le cas des apostrophes à connotation moins intime et plus «cool» comme 'miss', 'man' ou 'buddy', et qui apparaissent notamment dans les salutations en ouverture des messages, en combinaison soit avec le français (49-50) soit avec l'anglais (5153):

(49) Salut ptite miss, je ne viens pas au colloque, mais je te tiens les pouces! Allez (merde)... A tt bientot

(50) salut miss, on pourrait s'échanger les dates de nettoyage du théatre, je vais le samedi 28 et toi le dimanche 29 ? Biz

(51) Sorry man j'étais en train de boire un thé chez aurélie, on s'appelle demain? Toute toute mec.

(52) Ya man,merci.je serai a l'arret d charmettes.bis dænn

(53) Hey buddy! On se fait une soirée film + chips demain soir chez toi à laus. ? ((angl.: 'le copain'))

Les termes cités se retrouvent à l'oral spontané dans le parler des jeunes, où notamment 'man' et 'miss' sont sédimentés. En français parlé informel des jeunes, le terme 'miss' (voir note 4 supra), par exemple, est utilisé dans les ouvertures («salut miss»), mais aussi comme synonyme de 'nana' ou 'jeune fille' dans des énoncés du type «j'ai vu une miss l'autre jour».

En somme, dans les messages SMS de notre corpus, l'alternance codique se trouve régulièrement associée à des apostrophes aussi bien tendres que «branchées». Typiquement, celles-ci figurent au début ou vers la fin des messages et sont liées à des salutations ou à des adieux. Ces apostrophes couvrent un éventail de formes, allant de formules internationalisées, régulièrement produites en anglais, en italien ou en espagnol, à des formes plus idiosyncrasiques, utilisées notamment pour des petits noms tendres ou humoristiques. L'anglais semble être la langue exclusive pour les formules «branchées» de type 'man' ou 'buddy', alors que l'italien et l'espagnol apparaissent, tout comme l'anglais, dans des apostrophes à connotation affective ('bella'). Pour ces dernières, toutefois, c'est le français plutôt que l'alternance codique qui représente la solution typique: dans le corpus, des apostrophes comme 'chouchou', 'poulet', ou 'mon cœur' abondent. De manière intéressante, l'allemand ou le suisse-allemand ne sont qu'exceptionnellement utilisés pour des apostrophes (comme par ailleurs pour l'expression de l'affectivité), ce qui suggère que ces langues tendent à jouer un rôle différent de l'anglais, l'italien et l'espagnol dans la communication par SMS du moins chez des sujets qui ne semblent pas être bilingues français-allemand/suisseallemand -, un rôle peut-être moins associé à l'expression de la proximité.

\subsection{Des alternances codiques pour indexer le lieu du destinateur/du destinataire}

Un dernier emploi de l'alternance codique, différent des emplois discutés jusqu'ici et moins fréquent, est de nature quasi-déictique dans le sens où il indique le lieu présent ou passé du 
destinateur ou du destinataire. Cela est rendu explicite dans l'extrait (54) dont l'auteur déclare, en anglais, qu'il se trouve à New York au moment où il rédige le message.

(54) Hi baby! Sorry I'm in New York City at the moment! Hihi! Je te raconterai! ((angl.: 'salut bébé. Excuse je suis à New York en ce moment'))

En (55), l'emploi de l'italien indexe ici encore - mais de manière moins explicite - le lieu de séjour de l'auteur au moment de la rédaction du message (voir également l'ex. 28 supra):

(55) Ciao della Sardegna! Alors ça été c'te lecture jeudi passé ?

((it.: 'salut de la Sardaigne'))

Par contre, en (56) c'est le lieu de séjour antérieur du destinataire qui est évoqué par l'utilisation de l'allemand, et la mention explicite de 'Berlin':

(56) Salut mousse ! ça va ? Wie war es im Berlin ?? Ah j'aurais tellement voulu être là

((all.: 'comment c'était à Berlin'))

pour ça!!

On notera par ailleurs en (55) que la forme appropriée en langue cible serait 'Ciao dalla Sardegna' (et non pas 'della') et en (56) 'Wie war es in Berlin' (et non pas 'im'), ce qui pourrait indiquer que les auteurs de ces messages ne maîtrisent pas particulièrement bien les langues mobilisés pour l'alternance codique - et suggère en plus qu'ils ne sont pas bilingues au sens classique du terme. Dans tous les cas cités, la langue utilisée dans le segment qui correspond à une alternance codique porte une valeur déictique dans le sens où elle renvoie aux lieux/environnements de l'énonciateur ou du destinataire et contribue ainsi à augmenter le partage expérientiel entre les interactants.

\section{Discussion}

La présente étude s'est proposée de contribuer à une meilleure compréhension de l'alternance codique dans la communication par SMS, en se focalisant sur un corpus de messages SMS recueillis en Suisse. Sur la base de la récurrence d'un nombre limité de formes pour un nombre limité d'actions types, les analyses ont identifié une série de caractéristiques formelles et fonctionnelles de l'alternance codique dans le corpus SMS. Les résultats, qui seront récapitulés dans ce qui suit, montrent que la présence régulière de l'alternance codique dans la communication par SMS ne se laisse pas résumer à des contraintes pratiques et techniques du SMS, dans une logique d'économie. Tout au contraire, elle relève de choix systématiques des utilisateurs de SMS, témoignant d'un maniement à toutes fins pratiques de codes autres que le français.

\subsection{Quelles formes d'alternance codique?}

La forme typique que prend l'alternance codique dans les données étudiées est celle de mots isolés ou d'expressions - pour la plupart en anglais - hautement internationalisées, et de ce fait transparentes. Ces alternances puisent fortement dans un éventail limité de formes récurrentes qui remplacent entièrement ou partiellement leur équivalents français. Celle-ci sont de deux types: a) des formes monolexicales isolées: 'hello', 'hi', 'hey', mais aussi 'sorry', 'please' ainsi que 'now' et 'today' et les apostrophes comme 'amore', 'bella', 'man', 'miss' ou 'buddy'; b) des expressions figées comme 'thank you', 'a domani' ou 'good luck'. Les alternances recensées sont le plus souvent des formules occupant un énoncé entier et ayant la valeur d'acte p.ex. de salutation ou de remerciement ('hello', 'thank you'); il s'agit de mots isolés ou expressions figées, et non pas de séquences structurées grammaticalement. C'est pour cette raison que nous les avons traitées comme des cas à part, différents de l'alternance entre deux langues de base (de type 'alternation'), mais aussi de l'insertion. Les alternances de type 'insertion' sont elles aussi récurrentes, alors que l'alternance entre deux langues de base 
('alternation') est plus rare et que les emprunts spontanés sont quasiment absents des données. De manière intéressante, les formes récurrentes de l'alternance codique ne relèvent le plus fréquemment pas de constituants propositionnels (nom ou $\mathrm{SN}$, verbe ou $\mathrm{SN}$, etc.). A l'exception des 'now' et 'today', il s'agit au contraire d'apostrophes et de formules à valeur de vœux, de remerciement, de bonjour ou d'adieu.

On constate en somme que les auteurs de messages SMS empruntent, notamment à l'anglais, un nombre limité de formules «prêtes à l'emploi», qui sont injectées telles quelles dans les messages, sans nécessiter ni compositions ni adaptations morpho-syntaxiques. L'une ou l'autre des expressions recensées se retrouve régulièrement en tant qu'emprunt sédimenté dans le langage parlé informel, notamment celui des jeunes (c'est le cas de 'miss', de 'ciao', mais aussi de 'hello'), mais la récurrence de la plupart d'entre elles semble être un trait spécifique de la communication par SMS.

\subsection{Quelles fonctions de l'alternance codique, et pour quelles langues?}

Dans les données étudiées, l'alternance codique a typiquement une fonction de démarcation. Elle met en relief des actions à valeur hautement phatique (salutations, excuses, vœux) et des témoignages d'affection ('I love you'), et elle est régulièrement associée aux apostrophes, allant des termes d'adresse à connotation «cool» comme 'man' ou 'buddy' à des petits noms tendres comme 'cookie'. Dans tous ces cas de figure, l'alternance codique, qui se trouve souvent combinée avec des points d'exclamation, et parfois avec des majuscules et des émoticônes, sert à augmenter l'expressivité du segment de discours concerné. En outre, ces emplois témoignent d'une distribution fonctionnelle tendancielle des langues: généralement, l'anglais domine, mais l'italien et l'espagnol interviennent à côté de l'anglais, en particulier pour l'expression de l'affectivité (cf. Cougnon, à paraitre, pour des observations similaires relatives à un corpus recueilli en Belgique). Mais, en raison de leur nature hautement routinisée (et internationalisée), les formules code-switchées se révèlent en quelque sorte comme des formules publiques, ce qui, du coup, attenue leur valeur interpersonnelle: un 'I love you' lancé en adieu communique-t-il la même chose, et est-il reçu de la même manière qu'un 'je t'aime'?

De manière quelque peu surprenante, étant donnée la situation linguistique de la Suisse, l'allemand et le suisse allemand sont plutôt rares; ils se retrouvent de manière régulière dans les alternances de type 'alternation', qui ne représentent que $12 \%$ des alternances recensées dans notre corpus et qui pourraient bien avoir été produites par des bilingues français(suisse)allemand (messages dont nous n'avons que marginalement tenu compte ici - et cela pour des raisons méthodologiques; cf. pt. 4.2 supra).

Un seul contexte récurrent a pu être identifié concernant l'alternance entre deux langues de base (au sens de la structuration grammaticale des énoncés) - ce qui est possiblement dû aux limites d'un corpus qui consiste en des messages isolées et ne permet pas de suivre des échanges de messages: ce type d'alternance sert régulièrement à indexer le lieu actuel ou passé du destinateur ou du destinataire ('Wie war es im Berlin'). Ici encore, l'alternance met en relief le segment concerné, contribuant en l'occurrence à augmenter le partage expérientiel entre les interactants en indexant un domaine d'expérience spécifique. Une telle pratique, toutefois, n'est pas unique à la communication par SMS; elle nous rappelle au contraire les habitudes classiques d'écriture de cartes postales (cf. Albert 1985).

Dans leur ensemble, ces résultats montrent que les alternances codiques dans les messages SMS ne relèvent pas d'opérations aléatoires, mais de choix qui sont instrumentaux dans la structuration de la dimension socio-interactive de la communication par SMS, et en particulier dans la gestion de la relation interpersonnelle. 
Ce point peut être étayé par le placement séquentiel de l'alternance au sein des messages. De manière intéressante, les données montrent une forte concentration des alternances en début et à la fin des messages. Ce phénomène ne se limite pas aux formules de salutation ou d'adieu, ni aux apostrophes, qui naturellement sont associées à ces positions séquentielles, mais se retrouve fréquemment pour d'autres utilisations d'une langue autre que le français:

(57) Help! Peux-tu donner les cours de nat cet aprèm de 14 à $17 \mathrm{~h} 30$ à Romanel? ((angl.: 'à l'aide'))

(58) Ach j'ai plus le mail en tete il manque clémence et ANON c'est les cornes et le briquet ((all.: particule exprimant une plainte)) et clé je sais plus!

(59) Of course frangine! Je me renseigne pour trouver un ordi qui convienne et je te tiens ((angl.: 'bien sûre')) au jus. Bon début de semaine.

(60) Anyway voici ma proposition: mets- moi sur le plan du 1 er janvier à ta place. ((angl.: 'quoi qu'i en soit')) Vraiment cela me conviens pour ce jour- là! Thanks, A

(61) Good news. Les cours commencent semaine 37 à la HEP. See you ((angl.: 'bonne nouvelle'))

((angl.: 'à bientôt'))

Il s'agit ici d'une caractéristique intéressante des messages. La relation intersubjective se jouant de manière particulièrement condensée dans l'ouverture et la clôture des échanges communicatifs, ce placement préférentiel des segments d'une autre langue que le français en début et en fin de message (qui ne correspondent pas forcément au début ou à la fin des échanges) met ici encore en relief une association privilégiée de l'alternance à des moments particulièrement pertinents pour le maintien de l'intersubjectivité.

\subsection{Un registre plurilingue spécifique aux SMS?}

Les formes et les fonctions de l'alternance codique dans les SMS s'inscrivent dans une logique de continuité par rapport au fonctionnement de l'alternance codique dans la communication verbale. Mais elles montrent également une série de traits distinctifs qui leur sont propres. L'alternance codique dans les SMS relève d'une routine communicative plurilingue qui semble différer en plusieurs points du «parler bilingue» documenté dans la communication en face-à-face. Parmi ces points on relèvera en premier lieu la mobilisation d'un nombre limité de formes récurrentes pour l'accomplissement d'un éventail limité de fonctions elles aussi récurrentes.

Sur le plan formel, le recours à une série d'expressions internationalisées dont le nombre est limité et qui proviennent en premier lieu de l'anglais suggère l'existence d'un type de pratique plurilingue spécifique à la communication par SMS (et peut-être plus généralement à la communication médiatisée par les nouvelles technologies) qui diffère justement des pratiques plurilingues au sens classique du terme - et cela auprès de sujets qui, eux (du moins pour la plupart), ne sont pas non plus plurilingues au sens classique du terme (voir Lüdi/Py 2003, pour une conception qui insiste sur le continuum entre pratiques uni- et plurilingues). Tout au contraire, il est possible que les résultats cités témoignent, du moins pour une part importante, d'une compétence plurilingue en filigrane auprès d'utilisateurs dont les pratiques hors communication par SMS ne sont pas plurilingues.

Sur le plan fonctionnel, les alternances suivent dans le fond les principes qui ont été décrits pour la communication en face-à-face (cf. pt. 2 supra). Elles fonctionnent comme des indices de contextualisation (Gumperz 1977), ayant notamment un effet de démarcation (pour la conversation ordinaire voir Auer 1984; Gumperz 1982; Lüdi/Py 2003; Li Wei 2002). On notera toutefois que leur inventaire fonctionnel est très restreint et qu'il repose sur des 
couplages récurrents forme-fonction, ce qui contraste fortement avec les pratiques diversifiées du code-switching attestées pour la communication orale. On notera également, dans les messages SMS, l'association régulière de l'alternance codique à l'accomplissement d'actions à haut degré phatique et à l'expression de l'affectivité - et donc l'utilisation de langues autres que la langue principale des messages pour cette fin. Ce constat va à l'encontre des résultats d'études sociolinguistiques classiques sur le code-switching au sein de communautés bi- ou plurilingues, selon lesquels le vernaculaire local relèverait typiquement d'un 'we-code', représentant la langue de la proximité et des contacts personnels, alors que la langue officielle (p.ex. le standard face au dialecte) représenterait la langue de la distance, des contacts publics, administratifs et professionnels. A l'heure actuelle nous ne disposons pas d'informations relatives aux données socio-biographiques des auteurs des messages ni à l'insertion des messages individuels dans des échanges de messages, qui nous permettraient d'approfondir ce point. L'association du code-switching à la gestion de la relation interpersonnelle, telle qu'elle se présente dans les données SMS, contraste également avec les résultats des investigations sur la communication médiatisée par ordinateur (p.ex. Durham 2003; Goldbarg 2009; Ho 2006; Warschauer/El Said/Zohry 2002). Ces dernières suggèrent que, dans les chats et dans les échanges email, les langues avec lesquelles les locuteurs s'identifient le plus sont associées à l'expression de pensées personnelles et à la discussion de thèmes d'un certain degré d'intimité, alors que l'anglais est avant tout lié à des thèmes techniques relatifs notamment à l'internet et à l'ordinateur. Rien de tel n'est observable dans les données SMS ici étudiées.

L'utilisation quelque peu typée de l'alternance codique dans la communication SMS auprès d'une communauté qui n'est pas plurilingue au sens classique du terme décrit les contours d'une pratique du code-switching qui affiche la nature différente de ce type de communication (cf. également l'étude de Deumert/Masinyana 2008, cité sous 3). En ce sens, l'alternance codique - non pas en tant que telle, mais sous les formes spécifiques qu'elle prend dans les données étudiées - peut être comprise comme partie intégrante d'une pratique SMS dont un trait distinctif est justement sa nature plurilingue spécifique. La pratique de l'alternance relèverait en ce sens d'un 'doing being an sms user' (pour paraphraser Sacks 1992: 'doing being ordinary'): la mobilisation d'un code plurilingue représenterait un moyen déployé par les utilisateurs de SMS pour se faire reconnaître en tant qu'utilisateurs ordinaires, réguliers de ce moyen de communication, comme appartenant à une communauté SMS dont les contours sont probablement vagues, et qui est sans doute structurée de manière différentielle en fonction notamment de l'âge des utilisateurs et de la nature de leurs contacts.

\section{Conclusion}

La communication par SMS mobilise de nouvelles routines conversationnelles et de nouvelles pratiques d'utilisation du code linguistique (Thurlow 2003), voire des codes linguistiques. La coprésence de plusieurs codes dans les messages SMS au sein d'une communauté en principe non-plurilingue (bien que certains de ses membres puissent l'être), est à ce jour largement restée inexplorée. Or, les analyses présentées dans cet article révèlent une pratique SMS éminemment plurilingue, dans laquelle le jeu avec les langues fait partie du «parler SMS».

La mobilisation récurrente d'éléments de plusieurs langues au sein des messages SMS relèvet-elle d'un répertoire plurilingue spécifiquement SMS? Est-elle le signe d'appartenance à une communauté globalisée, d'un 'we-code' des utilisateurs de SMS? Fait-elle partie des 'méthodes' (au sens de procédés systématiques) déployées par les utilisateurs pour manifester et accomplir leur appartenance au groupe, d'un 'doing being an SMS user'?

Alors que les résultats obtenus dans cette étude exploratoire pointent en ces directions, de futures recherches systématiques sur la communication par SMS dans une variété de contextes linguistiques, géographiques, culturels et sociaux, sont nécessaires pour apporter 
des réponses fondées. La mise en rapport de l'analyse des messages avec les données sociobiographiques des utilisateurs pourra s'avérer d'un intérêt particulier à cet égard. Sur la base des données analysées ici, et en accord avec les interprétations actuelles de la communication médiatisée par les nouvelles technologies, ma position personnelle serait de voir en celle-ci non pas un monde à part, mais un ensemble de pratiques qui, bien que dotées d'une série de spécificités, s'inscrivent dans la continuité d'autres pratiques communicatives, d'autres espaces de socialisation, d'autres jeux de langage $-\mathrm{y}$ inclus des pratiques, des espaces, des jeux plus classiques.

Les résultats cités ici rejoignent les études sur l'alternance codique en montrant que le choix linguistique est lié à des enjeux pratiques. Mais ils montrent également une pratique qui est différente de ce que nous connaissons du parler bilingue en face-à-face. Ils révèlent des sédimentations d'une série de formules récurrentes, hautement routinisées - sédimentations qui semblent être spécifiques à la communication par SMS. Ils font voir des pratiques plurilingues déployées dans les SMS par des personnes qui ne s'engagent pas forcément dans de telles pratiques ailleurs. Ils mettent en relief l'émergence de nouveaux espaces plurilingues, indiquant une transformation de la nature traditionnelle des échanges communicatifs qui déstabilise les frontières entre ce que nous considérons communément comme pratiques uniet plurilingues et comme personnes uni- et plurilingues.

A une époque où le contact entre les langues s'intensifie dans le mouvement général de la globalisation, où nos pratiques quotidiennes se pluralisent, où la présence de certaines langues est parfois perçue comme menaçante pour d'autres, le plurilinguisme mérite d'être interrogé dans tous ses états. Les données SMS offrent un accès privilégié à des pratiques communicatives quotidiennes. Leur analyse a le potentiel de contribuer à une connaissance plus exhaustive de la diversité des pratiques communicatives plurilingues qui, possiblement, ne sera pas sans amener un re-calibrage de certains concepts clés nous permettant de les appréhender.

\section{Bibliographie}

Albert, Jean-Jacques (1985): "Bonjour de Neuchâtel où il fait beau et chaud. Essai d'interprétation d'un corpus de cartes postales de vacances". TRANEL 8, 69-94.

Anis, Jacques (2002): "Communication électronique scripturale et formes langagières: chats et sms". In: Actes des Quatrièmes Rencontres Réseaux Humains/Réseaux technologiques, Poitiers: Université de Poitiers. http://rhrt.edel.univ-poitiers.fr/document.php?id=547 $<12 / 09 / 2010>$.

Auer, Peter (1984): Bilingual Conversation. John Benjamins: Amsterdam.

id. (1996): "Bilingual Conversation. Dix ans après". AILE 7: 9-34.

id. (1998): "Introduction: bilingual conversation revisited". In: id. (ed.): Code-Switching in Conversation: Language, Interaction and Identity. London, Routledge: 1-24.

Bautista, Maria Lourdes S. (2004): "Tagalog-English code switching as a mode of discourse". Asia Pacific Education Review 5: 226-233.

Blom, Jan-Petter/Gumperz, John J. (1972): "Social meaning in linguistic structure: Codeswitching in Norway". In Gumperz, John/Hymes, Dell (eds.): Directions in Sociolinguistics. New York, Rinehart and Winston: 407-434.

Carrier, Mark L./Benitez, Sandra Y. (2010): "The effect of bilingualism on communication efficiency in text messages (SMS)". Multilingua - Journal of Cross-Cultural and Interlanguage Communication 29/2: 167-183.

Chiluwa, Innocent (2008): "Assessing the Nigerianness of SMS text-messages in English". English Today 24/1: 51-56.

Crystal, David (2008): Txtng: The gr8 db8. Oxford: Oxford University Press. 
Cougnon, Louise-Amélie (à paraitre): "'Tu te prends pour the king of the world?'. Language contact in text messaging context". In: Hasselblatt, Cornelius/Houtzagers, Peter/van Pareren, Remco (eds.): Language Contact in Times of Globalization. Amsterdam/New York, Rodopi: 45-59.

Cougnon, Louise-Amélie (2008): "Le français de Belgique dans 'l'écrit spontané'. Approche d'un corpus de 30.000 SMS". Travaux du Cercle Belge de Linguistique. http://webh01.ua.ac.be/linguist/SBKL/sbk12008/cou2008.pdf <10/06/2010>.

Danet, Brenda/Herring, Susan C. (2003): "Introduction: The Multilingual Internet. Journal of Computer Mediated Communication 9/1. http://jcmc.indiana.edu/vol9/issue1/intro.html $<20 / 09 / 2009>$.

eadem (2007): "Introduction". In: Danet, Brenda/Herring, Susan C. (eds.): The Multilingual Internet: Language, Culture, and Communication Online. New York, Oxford University Press: 3-39.

Da Silva, Samuel/Ndabihonge, Alexis/Schard, Emanuel (2009): "Etude de l'alternance codique dans un corpus de SMS en français". Travail de séminaire, Université de Neuchâtel.

Deumert, Ana, /Masinyana, Sibabalwe O. (2008): "Mobile language choices - The use of English and isiXhosa in text messages (SMS)". English World-Wide 29: 117-147.

Durham, Mercedes (2003): "Language choice on a Swiss mailing list". Journal of Computer Mediated Communication 9/1. http://jcmc.indiana.edu/vol9/issue1/durham.html $<20 / 09 / 2009>$.

Dürscheid, Christa/Stark, Elisabeth 2011): "SMS4science: An international corpus-based texting project and the specific challenges for multilingual Switzerland". In: Crispin Thurlow/Mroczek, Kristine (eds.): Digital Discourse. Language in the New Media. Oxford, Oxford University Press: 299-320.

Eaton, Lucas/Yarmanova, Elena (2009): "Code-switching for nicknames in SMS communication". Assignment paper, University of Luxemburg.

Fairon, Cédric/Klein, Jean/Paumier, Sébastien (2006): "Le langage sms: révélateur d'1compétence". Le français m'a tuer 1: 33-42.

Gafaranga, Joseph (2005): "Demythologising language alternation studies: conversational structure vs. social structure in bilingual interaction". Journal of Pragmatics 37: 281-300.

Global-Key Telecom, Mobile and Broadcast Statistics (2010). http://www.budde.com.au/ Research/Global-Key-Telecoms-Mobile-and-Broadband-Statistics.html <13/10/2010>.

Goldbarg, Rosalyn Negrón (2009): "Spanish English code switching in Email communication". Language @ internet 6. http://www.languageatinternet.org /articles/2009/2139<12/09/2010>.

Gumperz, John J. (1977): "Sociocultural knowledge in conversational inference. Linguistics and anthropology". In: Georgetown University Round Table on Languages and Linguistics 1977. Washington, D.C., Georgetown University Press: 191-211.

Gumperz, John J. (1982): Discourse Strategies. Cambridge: Cambridge University Press.

Haggan, Madeline (2007): "Text messaging in Kuwait. Is the medium the message"? Multilingua 26: 427-49.

Ho, Judy Woon Yee (2006): "Functional complementarity between two languages in ICQ". International Journal of Bilingualism 10/4: 429-421.

Li, Wei (1998): "The 'why' and 'how' questions in the analysis of conversational code switching". In: Auer, Peter (ed.): Code switching in conversation: Language, interaction and identity. London, Routledge: 156-176.

id. (2002): "'What do you want me to say?' On the Conversation Analysis approach to bilingual interaction". Language in Society 31, 150-180.

id. (2005): "'How can you tell?' Towards a common sense explanation of conversational code switching". Journal of Pragmatics 37/3: 375-389. 
Lüdi, Georges (2004): "Pour une linguistique de la compétence du locuteur plurilingue". Revue française de linguistique appliquée 9/2: 125-135.

Lüdi, Georges/Py, Bernard (2003 [1986]): Etre bilingue. Bern: Lang.

Mondada, Lorenza/Pekarek Doehler, Simona (2003): "Le plurilinguisme en action". In: Mondada, Lorenza/Pekarek Doehler, Simona (eds.): Plurilinguisme - Mehrsprachigkeit Plurilingualism. Enjeux identitaires, socio-culturels et éducatifs. Tübingen, Francke: 95110.

Muysken, Peter (2000): Bilingual speech: A typology of code mixing. Cambridge: Cambridge University Press.

Myers-Scotton, Carol (1992): "Comparing code switching and borrowing". In: Eastman, Carol M. (ed.): Codeswitching. Exeter, Multilingual Matters: 19-41.

Poplack, Shana (1980): "Sometimes I'll start a sentence in Spanish y termino en español: toward a typology of code-switching". Linguistics 18: 581-618.

Riegel, M./Pellat, J.-C./Rioul, R. (1994): Grammaire méthodique du français. Paris: PUF.

Sacks, Harvey (1992): Lectures on conversation. Oxford: Blackwell.

Sebba, Mark/Wootton, Anthony (1998): "We, they and identity: sequential vs. identity-related explanation in codeswitching". In: Auer, Peter (ed.): Code-Switching in Conversation: Language, Interaction and Identity. Routledge, London: 262-286.

[Oesch-] Serra, Cecilia (1992): "Code-switching et marqueurs discursifs: entre variation et conversation". TRANEL 18: 155-171.

Thurlow, Crispin (2003): "Generation Txt? The sociolinguistics of young people's textmessaging". Discourse Analysis Online 1/1: 1-27.

Thurlow, Crispin/Poff, Michele (à paraitre). "The language of text-messaging". In: Herring, Susane C./Stein, Dieter/Virtanen, Tuija (eds): Handbook of the Pragmatics of CMC. Berlin/New York: Mouton de Gruyter.

Warschauer, Mark/Said, Ghada R./Zohry, Ayman (2002): "Language choice online: Globalization and identity in Egypt". Journal of Computer Mediated Communication 7/4. http://jcmc.indiana.edu/vol7/issue4/warschauer.html <20/09/2010>. 